\title{
Avaliação in vitro da atividade antioxidante do extrato hidroalcoólico de folhas de bardana
}

\author{
Adriene R. Lima, Valéria C. Barbosa, Plínio R. Santos Filho, Cibele M.C.P.Gouvêa* \\ Departamento de Ciências Biológicas, Universidade Federal de Alfenas, Rua Gabriel Monteiro da Silva, 714, \\ Centro, 37130-000, Alfenas, MG, Brasil
}

\begin{abstract}
RESUMO: A atividade antioxidante do extrato hidroálcoólico de folhas de bardana (EEB), das frações acetato de etila (ACE) e hexano (HEX) foi avaliada por meio de testes in vitro. O EEB e frações inibiram a peroxidação lipídica em homogeneizado de cérebro de rato, com $\mathrm{IC}_{50}$ de 0,136 $\pm 0,015 ; 0,218 \pm 0,049$ e $0,628 \pm 0,092 \mathrm{mg} / \mathrm{mL}$ para o EEB, ACE e HEX respectivamente. O EEB, $\mathrm{ACE}$ e HEX apresentaram atividade seqüestrante de radicais $\mathrm{DPPH}$, com $\mathrm{IC}_{50}$ de $0,029 \pm 0,006$; $0,089 \pm 0,003$ e $0,837 \pm 0,160 \mathrm{mg} / \mathrm{mL}$ respectivamente. A capacidade antioxidante total do EEB foi significativamente maior $(\mathrm{p}<0,001)$ que a das frações sendo de 267,$20 ; 55,49$ e $50,02 \mathrm{mM}$ de ácido ascórbico, respectivamente, para o EEB, ACE e HEX. O EEB apresentou 7,88 $\pm 0,25 \%(\mathrm{~m} / \mathrm{m})$ de compostos fenólicos, que foi significativamente $(\mathrm{p}<0,001)$ diferente das ACE e HEX. Os resultados indicam que os extratos analisados apresentam atividade antioxidante, sendo que o EEB foi o mais eficiente. Este é o primeiro trabalho demonstrando a atividade antioxidante de folhas de bardana.
\end{abstract}

Unitermos: Arctium lappa, Asteraceae, antioxidante, TBARS, DPPH.

\begin{abstract}
In vitro evaluation of the antioxidant activity of the hydroalcoholic extract of leaves of bardana". The antioxidant activity of the hydroalcoholic extract of leaves of bardana (EEB), the ethyl acetate (ACE) and hexane (HEX) fractions were evaluated by in vitro assays. The EEB and fractions inhibited the lipid peroxidation in rat brain homogenate, with $\mathrm{IC}_{50}$ of $0.136 \pm$ $0.015 ; 0.218 \pm 0.049$ and $0.628 \pm 0.092 \mathrm{mg} / \mathrm{mL}$ for BEE, EAC and HEX respectively. The EEB, ACE and HEX presented DPPH radical scavenging-activity, with $\mathrm{IC}_{50}$ of $0.029 \pm 0.006 ; 0.089$ \pm 0.003 and $0.837 \pm 0.160 \mathrm{mg} / \mathrm{mL}$ respectively. The total antioxidant capacity of the EEB was significantly $(\mathrm{p}<0.001)$ higher than the fractions, being $267.20 ; 55.49$ and $50.02 \mathrm{mM}$ of ascorbic acid, respectively for EED, ACE and HEX. The ethanol extract presented $7.88 \pm 0.25 \%$ (w/w) of phenolic compounds that was significantly $(p<0.001)$ different from the ACE and HEX fractions. The results indicate that the analyzed extracts present antioxidant activity, and the EEB was the most efficient. This is the first report on the antioxidant activity of bardana leaves.
\end{abstract}

Keywords: Arctium lappa, Asteraceae, antioxidant, TBARS, DPPH.

\section{INTRODUÇÃO}

A bardana (Arctium lappa Linne) é uma planta perene, originária da Europa, pertencente à família Asteraceae. Esta planta é conhecida mundialmente e propaga-se espontaneamente por todo o Brasil. Caracteriza-se por apresentar folhas grandes e flores arroxeadas, podendo chegar a 2,5 metros de altura. É também conhecida como erva-dos-tinhosos, pegamassa, capricho-de-carneiro e capricho-grande (Holetz et al., 2002).

No Brasil é cultivada em todo o território, tem crescimento vigoroso, sendo considerada uma espécie daninha em pomares e terrenos baldios na região sul. Antigamente era utilizada em mistura com outras ervas, para clarear a pele e tem hoje aplicações como depurativo e antiinflamatório (Holetz et al., 2002). Tem sido cultivada há muito tempo no Japão e Taiwan, onde é consumida in natura (Morita et al, 1993) e utilizada como bebida para hipertensão, gota, arteriosclerose, hepatite e outras desordens inflamatórias (Lieber, 1994). As raízes são utilizadas, popularmente, como diurético e antipirético (Lin et al., 2002). A infusão das folhas também é utilizada com a mesma finalidade (Grases et al., 1994).

Trabalhos têm demonstrado que a bardana inibe a enzima conversora de angiotensina (Barbosa-Filho et al., 2006). Também, apresenta efeito hepato-protetor na administração crônica de etanol, contra carcinógenos e na prevenção de tumorigênese, provavelmente por apresentar lignanas com atividade antioxidante (Morita et al., 1984; Lin et al., 1996; Lin et al., 2000). Lin et al. (1996) relataram que $A$. lappa tem atividade antiinflamatória e seqüestrante de radicais livres. Tais efeitos têm sido atribuídos a alguns compostos predominantes em raízes e frutos da planta, como lignanas arctigenina (Umehara et al., 1996) e arctiina (Hirose et al., 2000). Wang e Yang (1993) relataram também a presença de daucosterol, matairesinol e lapaol em raízes e frutos. A importância dessa planta levou sua inclusão na Farmacopéia Brasileira primeira edição (Brandão et al., 2006). 
Os estudos prévios utilizam raízes e frutos de bardana e não há trabalhos demonstrando se as folhas de bardana apresentam atividade antioxidante. Assim, o presente trabalho teve como objetivo preparar e fracionar o extrato hidroalcoólico de folhas de bardana para avaliação, in vitro, da sua atividade antioxidante.

\section{MATERIAL E MÉTODOS}

\section{Preparo e fracionamento do extrato de bardana}

As folhas de bardana (Arctium lappa L., Asteraceae), coletadas em julho de 2005, no horto florestal da Universidade Federal de Lavras, foram secas em estufa com circulação de ar a $30^{\circ} \mathrm{C}$. A seguir, foram utilizadas $100 \mathrm{~g}$ de folhas secas para extração com etanol, por percolação, empregando-se uma mistura hidroalcóolica a $70 \%$ (v/v) (5 vezes de 20 min cada). O material foi filtrado e o filtrado foi concentrado em evaporador rotatório sob pressão reduzida, à temperatura de até $50{ }^{\circ} \mathrm{C}$, até o volume final de $100 \mathrm{~mL}$, obtendo-se o extrato etanólico bruto de bardana (EEB). Uma fração hexano (HEX) foi preparada por partição com n-hexano. A $50 \mathrm{~mL}$ de EEB foi adicionado $n$-hexano, em funil de separação, até a fase hexânica apresentar-se incolor. A fase hidroalcoólica resultante do processo anterior foi, então, particionada com acetato de etila em funil de separação, até que a fase acetato de etila se apresentasse incolor, resultando na fração acetato de etila (ACE) (Gegenheimer, 1990). $\mathrm{O}$ EEB e frações foram liofilizados. Para a utilização nos testes de atividade antioxidante, o EEB liofilizado foi ressuspenso em água destilada ou solução salina e as frações em DMSO a $1 \%(\mathrm{v} / \mathrm{v})$.

\section{Determinação in vitro da peroxidação de lipídios de cérebro de ratos}

A peroxidação lipídica foi determinada em homogeneizado de cérebro de rato, avaliando-se a formação de substâncias reativas com ácido tiobarbitúrico (TBARS), como descrito por Buege e Aust (1978). Foram utilizados 15 ratos machos Wistar, com $270 \pm 20 \mathrm{~g}$, obtidos no Biotério da UNIFAL-MG. O trabalho está de acordo com os princípios éticos na experimentação animal adotados pelo Colégio Brasileiro de Experimentação Animal (COBEA), tendo sido aprovado pela Comissão de Ética na Experimentação Animal (CEEA) da UNIFALMG, em reunião no dia 8 de março de 2004.

O homogeneizado de cérebro foi preparado como descrito por Paula et al. (2005), com modificações. $\mathrm{O}$ cérebro foi coletado, pesado e homogeneizado em 3 volumes de PBS 0,1 M, pH 7,4, contendo 3,5-diterc-butil-4-hidróxi tolueno (BHT) 0,06\% (m/v). Após a centrifugação da amostra, a $10.000 \mathrm{~g}$, por $15 \mathrm{~min}$, o sobrenadante foi coletado e a concentração de proteínas das amostras foi determinada pelo método de Peterson (1977). Alíquotas de homogeneizado contendo $2 \mathrm{mg}$ de proteínas foram colocadas em tubos de ensaio com concentrações diferentes do EEB e frações, separadamente. $\mathrm{O}$ volume das amostras foi completado para $1 \mathrm{~mL}$ com PBS e estas foram incubadas a $37^{\circ} \mathrm{C}$, por $30 \mathrm{~min}$. A seguir foram adicionados $0,5 \mathrm{~mL}$ de ácido clorídrico a $25 \%(\mathrm{v} / \mathrm{v})$ e $0,5 \mathrm{~mL}$ de ácido tiobarbitúrico a $1 \%(\mathrm{p} / \mathrm{v})$. A mistura foi aquecida em água fervente por 15 min e resfriada em banho de gelo por $10 \mathrm{~min}$. Foram acrescentados $2 \mathrm{~mL}$ de butanol e os tubos foram agitados vigorosamente em vórtex. Após a centrifugação a 10.000 $g$, por $15 \mathrm{~min}$ as TBARS foram determinadas por $\mathrm{Abs}_{535}$, utilizando-se o malonaldeído (MDA) como padrão $\left(\varepsilon=1,56 \cdot 10^{5} \mathrm{M}^{-1} \cdot \mathrm{cm}^{-1}\right)$. O EEB e frações incubados sem homogeneizado de cérebro foram utilizados como branco, para cada concentração testada. Os resultados foram expressos como $\eta \mathrm{mol} \mathrm{MDA} / \mathrm{mg}$ proteína em cada amostra. A atividade antioxidante foi calculada como porcentagem de inibição da peroxidação de lipídeos, segundo a equação:

Inibição da peroxidação lipídica

$$
(\%)=\left(\frac{C c-C t}{C c}\right) \times 100,
$$

Onde, Cc é a concentração de MDA do controle, que não foi incubado com o EEB e frações e $\mathrm{Ct}$ a concentração nas amostras incubadas com diferentes concentrações do EEB e frações.

A concentração do extrato $(\mathrm{mg} / \mathrm{mL})$ necessária para inibir $50 \%$ da peroxidação lipídica $\left(\mathrm{IC}_{50}\right)$ foi calculada.

\section{Atividade seqüestrante de radicais DPPH}

A atividade seqüestrante de radicais DPPH $(1,1$ difenil-1,2-picril hidrazil) foi determinada de acordo com o método de Yen e Wu (1999), com modificações. Concentrações diferentes dos EEB e frações foram adicionadas a $200 \mu \mathrm{L}$ de solução etanólica de DPPH $(0,5 \mathrm{mM})$ e o volume final foi ajustado para $1 \mathrm{~mL}$. A preparação foi incubada no escuro, sob temperatura ambiente por 30 min. Decorrido o tempo, a absorbância de cada solução foi determinada a $517 \mathrm{~nm}$. As atividades seqüestrantes de DPPH do EEB e frações foram expressas em porcentagem, segundo a equação:

Atividade seqüestrante de DPPH

$$
(\%)=100-\left[\left(\frac{A c-A t}{A c}\right) \times 100\right]
$$

Onde, Ac é a absorbância do controle, que não foi incubado com o EEB e frações e At a absorbância das amostras incubadas com diferentes concentrações do EEB e frações.

A concentração do extrato $(\mathrm{mg} / \mathrm{mL})$ necessária para atingir $50 \%$ de atividade seqüestrante de radicais $\mathrm{DPPH}\left(\mathrm{IC}_{50}\right)$ foi calculada. 


\section{Capacidade antioxidante total}

A capacidade antioxidante total foi determinada pelo método do fosfomolibdênio, que se baseia na determinação espectrofotométrica da redução do $\mathrm{Mo}^{+4}$ a $\mathrm{Mo}^{+5}$, com formação subseqüente de fosfato de $\mathrm{Mo}^{+5}$, que apresenta absorção máxima a $695 \mathrm{~nm}$ (Prieto et al., 1999). Alíquotas de $0,1 \mathrm{~mL}$ dos extratos $(10 \mathrm{mg} / \mathrm{mL})$ dissolvidas em água destilada (EEB) ou DMSO a 1\% (frações) foram combinadas, em tubo eppendorf, com 1 $\mathrm{mL}$ da solução reagente (ácido sulfúrico $600 \mathrm{mM}$, fosfato de sódio $28 \mathrm{mM}$ e molibdato de amônio $4 \mathrm{mM}$ ). Os tubos foram fechados e incubados a $95{ }^{\circ} \mathrm{C}$ por $90 \mathrm{~min}$. Após resfriamento, à temperatura ambiente, foi determinada a absorbância a 695 nm. Foi utilizado ácido ascórbico como padrão e a capacidade antioxidante total foi expressa em equivalentes de ácido ascórbico.

\section{Determinação de fenólicos totais}

Aconcentração de fenólicos totais dos extratos foi determinada como descrito por Woisk e Salatino (1998). Amostras de 0,1 g do EEB e frações foram misturadas com $0,5 \mathrm{~mL}$ de reagente Folin-Ciocalteu diluído em água (1:10). Depois de $8 \mathrm{~min}$, foram adicionados $0,4 \mathrm{~mL}$ de solução de carbonato de sódio a $4 \%(\mathrm{~m} / \mathrm{v})$ e os tubos foram mantidos no escuro à temperatura ambiente por 2 h. Decorrido o tempo a absorbância foi determinada a 740 $\mathrm{nm}$. A concentração de fenólicos foi calculada utilizandose ácido gálico como padrão.

\section{Análise estatística}

Os dados obtidos para os experimentos realizados representam a média seguida do erro padrão da média (EPM) e foram comparados por análise de variância pelo teste ANOVA, seguido do teste de Tukey-Kramer, quando $\mathrm{p}<0,05$.

\section{RESULTADOS}

\section{Peroxidação lipídica em cérebro de ratos}

Os resultados mostraram que o EEB e frações diminuíram a formação de MDA, indicando que houve inibição da peroxidação lipídica em cérebro de ratos. A inibição máxima obtida foi de 84,00 \% com o EEB; 82,82 $\%$ com a fração ACE e de $67,74 \%$ com a HEX e a $\mathrm{IC}_{50}$ foi de $0,136 \pm 0,015 ; 0,218 \pm 0,049$ e $0,628 \pm 0,092 \mathrm{mg} / \mathrm{mL}$, respectivamente (Figura 1).

\section{Atividade seqüestrante de radicais DPPH}

O EEB e frações demonstraram atividade seqüestrante de radicais DPPH. O EEB apresentou atividade significativamente maior que as frações $(\mathrm{p}<0,05)$. A atividade máxima para o EEB foi de 90,79
\%; para a fração ACE foi de 90,05 \% e para a HEX foi de $87,98 \%$ e a $\mathrm{IC}_{50}$ foi de $0,029 \pm 0,006 ; 0,089 \pm 0,003$ e $0,837 \pm 0,160 \mathrm{mg} / \mathrm{mL}$ respectivamente (Figura 2 ).

\section{Capacidade antioxidante total}

A capacidade antioxidante total do extrato foi significativamente maior $(p<0,001)$ que a das frações. A capacidade antioxidante total do EEB, ACE e HEX foi, respectivamente, de 267,20; 55,49 e 50,02 mM de ácido ascórbico (Tabela 1).

\section{Fenólicos totais}

O EEB apresentou 7,88 $\pm 0,25 \%(\mathrm{~m} / \mathrm{m})$ de compostos fenólicos, que foi significativamente $(\mathrm{p}<0,001)$ diferente das frações ACE e HEX (Tabela 1).

\section{DISCUSSÃO}

Desde o início da vida no planeta, a toxicidade do oxigênio atmosférico tem sido o principal desafio à sobrevivência dos organismos vivos. $\mathrm{O}_{2}$ participa de reações de óxido-redução e é o aceptor final de $\mathrm{H}^{+}$ no metabolismo normal de mamíferos. As oxidações biológicas geram espécies reativas de oxigênio e radicais livres que podem provocar lesões celulares, muitas vezes irreparáveis. O estresse oxidativo leva a danos a lipídeos, DNA, proteínas e organelas celulares, como mitocôndria e membranas, provocando alterações da estrutura e função celulares, que levam ao desenvolvimento de câncer, envelhecimento precoce, doenças cardiovasculares, degenerativas, neurológicas entre outras. A produção contínua de radicais livres durante os processos metabólicos levou ao desenvolvimento de muitos mecanismos de defesa antioxidante responsáveis pela inibição e redução das lesões causadas pelos radicais livres nas células (Gouvêa, 2004).

Os antioxidantes não-enzimáticos incluem o ácido ascórbico, vitamina E, carotenóides, compostos fenólicos e polifenólicos, além de outros (Bianchi e Antunes, 1999). A atividade antioxidante de compostos vegetais pode ser atribuída a vários mecanismos, como a prevenção da peroxidação lipídica e capacidade sequëstrante de radicais livres (Kaur; Geetha, 2006).

$\mathrm{O}$ extrato aquoso, etanólico e metanólico de raízes e frutos de bardana têm demonstrado atividade antioxidante (Lin et al., 1996; Lin et al., 2000; 2002). Contudo, a atividade antioxidante das folhas ainda não está estabelecida. Neste estudo avaliou-se a atividade antioxidante do extrato hidroalcoólico de folhas de bardana (EEB) e frações, in vitro, abordagem mais indicada para verificação da atividade antioxidante potencial de um extrato vegetal.

Nesse trabalho verificou-se que o EEB e frações diminuíram a peroxidação lipídica. A lipoperoxidação é caracteristicamente uma reação em cadeia iniciada 


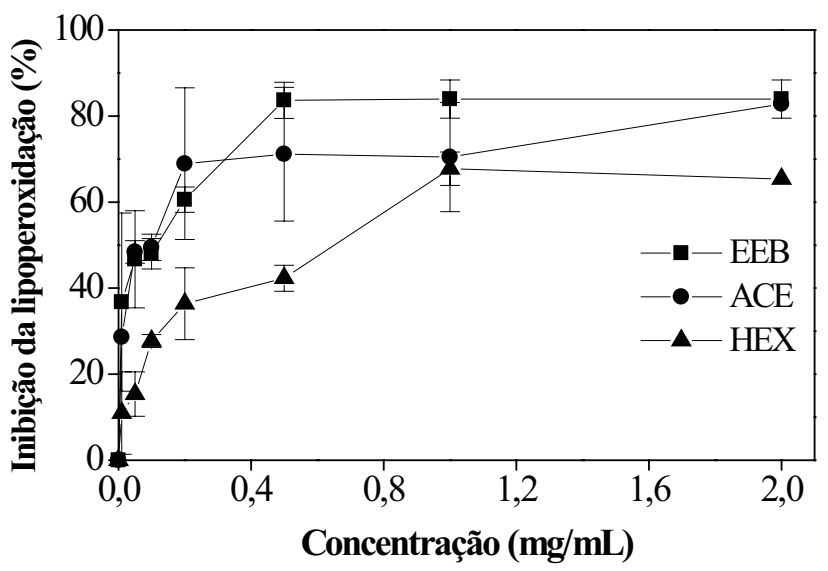

Figura 1. Porcentagem de inibição da peroxidação lipídica em cérebro de ratos do extrato hidroalcoólico de bardana (EEB), fração acetato de etila (ACE) e fração hexano (HEX). A $\mathrm{IC}_{50}$ foi de 0,136 $\pm 0,015 ; 0,218 \pm 0,049$ e $0,628 \pm 0,092 \mathrm{mg} / \mathrm{mL}$ para o EEB, ACE e HEX respectivamente. Os resultados representam a média \pm erro padrão da média $(\mathrm{n}=3)$.

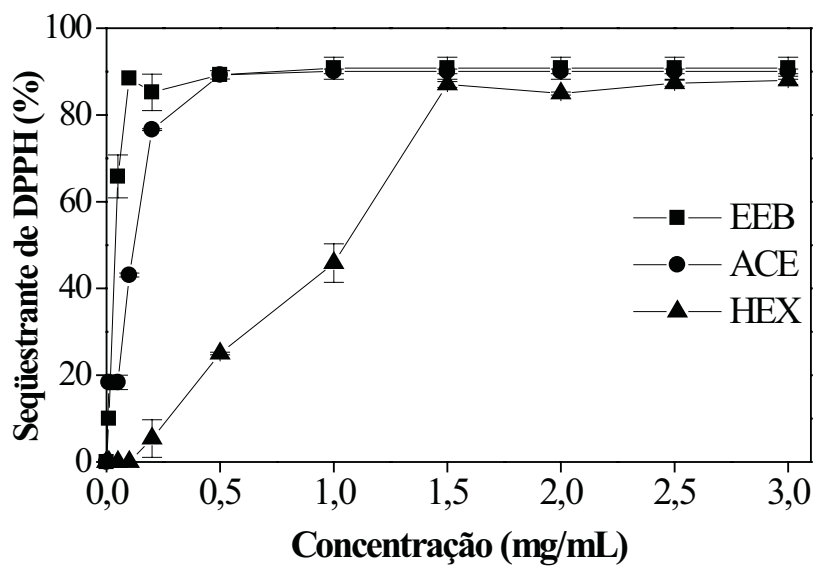

Figura 2. Atividade seqüestrante de radicais DPPH do extrato hidroalcoólico de bardana (EEB), fração acetato de etila (ACE) e fração hexano (HEX). A IC $\mathrm{I}_{50}$ foi de $0,029 \pm 0,006 ; 0,089 \pm 0,003 \mathrm{e}$ $0,837 \pm 0,160 \mathrm{mg} / \mathrm{mL}$ para o EEB, ACE e HEX respectivamente. Os resultados representam a média \pm erro padrão da média $(n=3)$.

Tabela 1. Capacidade antioxidante total e conteúdo de compostos fenólicos do extrato hidroalcoólico de bardana (EEB), da fração acetato de etila (ACE) e fração hexano (HEX).

\begin{tabular}{lcc}
\hline Fração & $\begin{array}{c}\text { Capacidade antioxidante total } \\
(\mathrm{mM} \text { de ácido ascórbico) }\end{array}$ & $\begin{array}{c}\text { Fenólicos totais } \\
(\%, \mathrm{~m} / \mathrm{m})\end{array}$ \\
\hline EEB & $267,2^{\mathrm{a}}$ & $7,88 \pm 0,25^{\mathrm{a}}$ \\
ACE & $55,49^{\mathrm{b}}$ & $1,02 \pm 0,05^{\mathrm{b}}$ \\
HEX & $50,02^{\mathrm{b}}$ & $0,59 \pm 0,05^{\mathrm{b}}$ \\
\hline
\end{tabular}

Letras diferentes na mesma coluna indicam diferença significativa $(\mathrm{p}<0,001)$ pelo teste de Tukey-Kramer. 
pela abstração de um átomo de hidrogênio de um ácido graxo poliinsaturado por um radical livre. A peroxidação lipídica pode formar radicais que propagam a reação peroxidativa e carbonilas tóxicas como o malonaldeído, que é citotóxico e pode lesar o DNA (Esterbauer, 1993). Assim, o extrato de bardana pode ser um auxiliar na prevenção e combate aos danos celulares causados pela peroxidação lipídica.

A avaliação da capacidade seqüestrante de radicais DPPH mostrou que o EEB e frações apresentam atividade antioxidante, sendo o EEB mais potente. O mecanismo de redução de radicais livres DPPH envolve a doação de hidrogênio. Neste sistema tanto a estrutura planar como espacial do composto antioxidante é importante. Baseado em dados da literatura é possível inferir que a potente atividade antioxidante de extratos polares é dada pela presença de substâncias com hidroxilas (Harborne e Williams, 2001; Mensor et al., 2001). Os resultados deste trabalho indicam que o extrato de folhas de bardana e frações apresentam capacidade para doar hidrogênio, pois apresentaram atividade seqüestrante de DPPH.

Os compostos fenólicos presentes em vegetais têm recebido considerável atenção por serem os principais componentes com atividade antioxidante, embora não sejam os únicos. A atividade antioxidante de compostos fenólicos tem sido atribuída às suas propriedades de óxido-redução, que desempenham importante papel na adsorção ou neutralização de radicais livres (Basile et al., 2005). O EEB e frações apresentam compostos fenólicos, o que pode contribuir, pelo menos em parte, para a atividade antioxidante demonstrada neste estudo. O conteúdo de fenólicos foi maior no EEB, o que pode contribuir para atividade antioxidante mais potente que as frações. Ainda, a complexação do $\mathrm{Fe}^{+2}$ a compostos fenólicos pode reduzir a disponibilidade deste metal envolvido na reação de Fenton e que participa tanto na iniciação como na propagação da peroxidação lipídica. Dessa forma, o conteúdo de compostos fenólicos do EEB pode ter contribuído para o melhor efeito deste extrato na inibição da peroxidação lipídica, verificada no presente trabalho.

Concluindo, o extrato de folhas de bardana apresenta atividade antioxidante, podendo atuar como seqüestrante ou por meio da redução de radicais livres e inibição da peroxidação lipídica, o que pode contribuir para a prevenção ou redução do desenvolvimento de patologias associadas ao estresse oxidativo. Para que os dados do presente trabalho sejam confirmados é importante que seja realizado estudo in vivo com o EEB e posterior fracionamento do extrato para determinação das substâncias ativas.

\section{AGRADECIMENTOS}

Ao Prof. Dr. José Eduardo Brasil Filho da UFLA, por ter cedido as folhas de bardana.

\section{REFERÊNCIAS}

Barbosa-Filho JM, Martins VKM, Rabelo LA, Moura MD, Silva MS, Cunha EVL, Souza MFV, Almeida RN, Medeiros IA 2006. Natural products inhibitors of the angiotensin converting enzyme (ACE). A review between 19802000. Rev Bras Farmacogn 16: 421-446.

Basile A, Ferrara L, Del Pozzo M, Mele G, Sorbo S, Bassi P, Montesano D 2005. Antibacterial and antioxidant activities of ethanol extract from Paullinia cupana Mart. J Ethnopharmacol 102: 32-36.

Bianchi MLP, Antunes LMG 1999. Radicais livres e os principais antioxidantes da dieta. Rev Nutr 12: 123-130.

Brandão MGL, Cosenza GP, Moreira RA, Monte-Mor RLM 2006. Medicinal plants and other botanical products from the Brazilian Official Pharmacopoeia. Rev Bras Farmacogn 16: 408-420.

Buege JA, Aust SD 1978. Microsomal lipid peroxidation. Meth Enzymol 52: 302-310.

Esterbauer H 1993. Cytotoxicity and genotoxicity of lipidoxidation products. Am J Clin Nutr 57: 779S-785S.

Gegenheimer P 1990. Preparation of extracts from plants. Methods Enzymol 182: 174-193.

Gouvêa CMCP 2004. Oxidações biológicas e atividade vegetal. In: Fitoterápicos anti-inflamatórios: aspectos químicos, farmacológicos e aplicações terapêuticas. Carvalho, J.C.T. coord. Ribeirão Preto: Tecmedd, p. 101-124.

Grases F, Melero G, Costa-Bauza A, Prieto R, March JG 1994. Urolithiasis and phytotherapy. Int Urol Nephrol 26: 507-511.

Harborne JB, Williams CA 2001. Anthocyanins and other flavonoids. Nat Prod Rep 18: 310-333.

Hirose M, Yamaguchi T, Lin C, Kimoto N, Futakuchi M, Kono T, Nishibe S, Shirai T 2000. Effects of arctiin on PhIP-induced mammary, colon and pancreatic carcinogenesis in female Sprague-Dawley rats and MeIQx-induced hepatocarcinogenesis in male F344 rats. Cancer Lett 155: 79-88.

Holetz FB, Pessini GL, Sanches NR, Cortez DA, Nakamura CV, Filho BP 2002. Screening of some plants used in the Brazilian folk medicine for the treatment of infectious diseases. Mem I Oswaldo Cruz 97: 1027-1031.

Kaur IP, Geetha T 2006. Screening methods for antioxidants-a review. Mini Rev Med Chem 6: 305-312.

Lieber CS 1994. Alcohol and the liver: 1994 update. Gastroenterology 106: 1085-1105.

Lin SC, Chung TC, Lin CC, Uengh TH, Lin YH, Lin SY, Wang L 2000. Hepatoprotective effects of Arctium lappa on carbon tetrachloride- and acetaminophen-induced liver damage. Am J Chin Med 28: 163-173.

Lin SC, Lin CH, Lin CC, Lin YH, Chen CF, Chen IC, Wang LY 2002. Hepatoprotective effects of Arctium lappa Linne on liver injuries induced by chronic ethanol consumption and potentiated by carbon tetrachloride. J Biomed Sci 9: 401-409.

Lin CC, Lu JM, Yang JJ, Chuang SC, Ujiie T 1996. Antiinflammatory and radical scavenge effects of Arctium lappa. Am J Chin Med 24: 127-137.

Mensor LL, Menezes FS, Leitão GG, Reis AS, Santos TC, Coube CS 2001. Screening of Brazilian plant extracts for antioxidant activity by the use of DPPH free radical 
method. Phytother Res 15: 27-30.

Morita K, Kada T, Namiki M 1984. A desmutagenic factor isolated from burdock (Arctium lappa Linne). Mutat Res 129: 25-31.

Morita T, Ebihara K, Kiriyama S 1993. Dietary fiber and fatderivatives prevent mineral oil toxicity in rats by the same mechanism. J Nutr 123: 1575-1585.

Paula FBA, Gouvea CMCP, Alfredo PP, Salgado I 2005. Protective action of a hexane crude extract of Pterodon emarginatus fruits against oxidative and nitrosative stress induced by acute exercise in rats. BMC Complement Altern Med 5: 17

Peterson GL 1977. A simplification of the protein assay method of Lowry et al. which is more generally applicable. Anal Biochem 83: 346-356.

Prieto P, Pineda M, Aguilar M 1999. Spectrophotometric quantitation of antioxidant capacity through the formation of a phosphomolybdenum complex: specific application to the determination of vitamin E. Anal Biochem 269: 337-341.

Umehara K, Nakamura M, Miyase T, Kuroyanagi M, Ueno A 1996. Studies on differentiation inducers: VI. Lignan derivatives from Arctium fructus. Chem Pharm Bull 44: 2300-2304.

Wang HY, Yang JS 1993. Studies on the chemical constituents of Arctium lappa L. Yao Xиe Xиe Bao 28: 911-917.

Woisk RG, Salatino A 1998. Analisys of propolis: some parameters and procedures for chemical quality control. J Apicult Res 37: 99-105.

Yen G, WU J. 1999 Antioxidant and radical scavenging properties of extracts from Ganoderma tsugae. Food Chem 65: 375-379. 